\title{
Free convection inside a porous square cavity with convective boundary condition using spline functions
}

\author{
Sanda Micula ${ }^{1}$ and loan Pop ${ }^{*}$ (D)
}

\section{"Correspondence:}

popm.ioan@yahoo.co.uk; ipop@math.ubbcluj.ro

'Department of Mathematics,

Faculty of Mathematics and

Computer Science, Babeş-Bolyai

University, 400084 Cluj-Napoca, Romania

\section{Springer}

\begin{abstract}
In this paper, we consider the problem of free convection in a square cavity filled with a porous medium with convective boundary condition on the left wall of the cavity. We first transform the governing equations transformed in terms of dimensionless variables and then solve them numerically using a cubic spline colocation method. We discuss the effects of two very important parameters, the Biot (Bi) and Rayleigh $(R a)$ numbers. We perform a comparison of the average Nusselt number $\overline{N u}$ at the hot wall with results from the open literature. We can notice that the comparison is very good, which gives us strong confidence that this cubic spline collocation method works very efficiently for such problems. We also state that the present problem has not been considered before by any researcher.
\end{abstract}

Keywords: Square cavity; Free convection; Porous medium; Convective boundary condition; Spline collocation method

\section{Introduction}

Convection in enclosures saturated with porous media is present in several transport operations in engineering devices. This is advised for altitude heat-dissipating electronic ingredients, where free convection is notable for providing effective cooling, heat exchangers, solar energy storage, greasing technology, cooling the electronic systems, and desiccation technologies. It also occurs in investigations considering the chemical reactions and detaching fluids, food processing and storage, petroleum and geothermal industries, dialysis of blood in artificial kidney, blood oxygenators and flow of blood in the capillaries, fibrous insulation, thermal insulation of buildings, geophysical systems, solar power collectors, nuclear reactors, geothermal applications, electro chemistry, metallurgy, underground disposal of nuclear or nonnuclear waste, and so on (see the books by Ingham and Pop [1-3], Pop and Ingham [4], Kaviany [5], Vafai [6, 7], Vadasz [8], Bejan [9], Nield and Bejan [10], Bear [11], Prasad and Kulacki [12], and Gross et al. [13]).

The situation with convective boundary condition arises in conjugate convective flows, where the heat is supplied to the convective fluid through a bounding surface with a finite heat capacity. This configuration occurs in many important engineering devices, for ex-

(c) The Author(s) 2021. This article is licensed under a Creative Commons Attribution 4.0 International License, which permits use, sharing, adaptation, distribution and reproduction in any medium or format, as long as you give appropriate credit to the original author(s) and the source, provide a link to the Creative Commons licence, and indicate if changes were made. The images or other third party material in this article are included in the article's Creative Commons licence, unless indicated otherwise in a credit line to the material. If material is not included in the article's Creative Commons licence and your intended use is not permitted by statutory regulation or exceeds the permitted use, you will need to obtain permission directly from the copyright holder. To view a copy of this licence, visit http://creativecommons.org/licenses/by/4.0/. 
ample, in heat exchange where the conduction in the solid tube wall is greatly influenced by the convection in the fluid flowing over it (see Chaudhary and Jain [14]).

We also mention some very good papers on efficient methods for such problems: Abdelwahed et al. [15] on reconstruction of Tesla micro-valve using topological sensitivity analysis, Abdelwahed and Chorfi [16] on the convergence analysis of a time-dependent elliptic equations with discontinuous coefficients, and Chorfi et al. [17] on the analysis of a geometrically selective turbulence model.

The objective of the present analysis is investigating the influence of the thermal boundary condition in a square cavity filled with a porous medium using a spline colocation method. We determine numerical solutions for the streamlines, isotherms, and local and average Nusselt numbers.

\section{Mathematical formulation}

We consider the free convection in a square cavity filled with a fluid-saturated porous medium, as it is shown in Fig. 1 , where the Cartesian coordinates are $\bar{x}$ and $\bar{y}$, and $L$ is the wall length. The temperatures at the left and right sides of the walls are considered constant, and the bottom and top walls are thermally insulated. We also assume that the outside left side surface of the cavity is heated by convection from a hot fluid at constant temperature $T_{f}$, which provides a heat transfer coefficient $h_{f}$ (see Aziz [18]), namely

$$
-k_{f} \frac{\partial T}{\partial \bar{y}}=h_{f}\left(T_{f}-T\right) \quad \text { on } y=0 .
$$

The homogeneity and the thermal equilibrium of the porous medium are assumed.

Then the steady conservation of mass, Darcy's law with the Oberbeck-Boussinesq approximation employed, and the energy equations are (see Nield and Bejan [10])

$$
\begin{aligned}
& \nabla \cdot \overline{\boldsymbol{v}}=0, \\
& 0=-\nabla p-\frac{\mu}{K} \overline{\boldsymbol{v}}+\rho_{0}\left[1-\beta\left(T-T_{c}\right)\right] \boldsymbol{g}, \\
& (\overline{\boldsymbol{v}} \cdot \nabla) T=\alpha_{m} \nabla^{2} T .
\end{aligned}
$$

In these equations, $\overline{\mathbf{v}}=(\bar{u}, \bar{v})$ is the velocity vector, $T$ is the fluid temperature, $p$ is the fluid pressure, $k$ is the thermal conductivity, $\nabla^{2}$ is the Laplace operator, and the physical meanings of the other quantities are mentioned in the Abbreviations list.

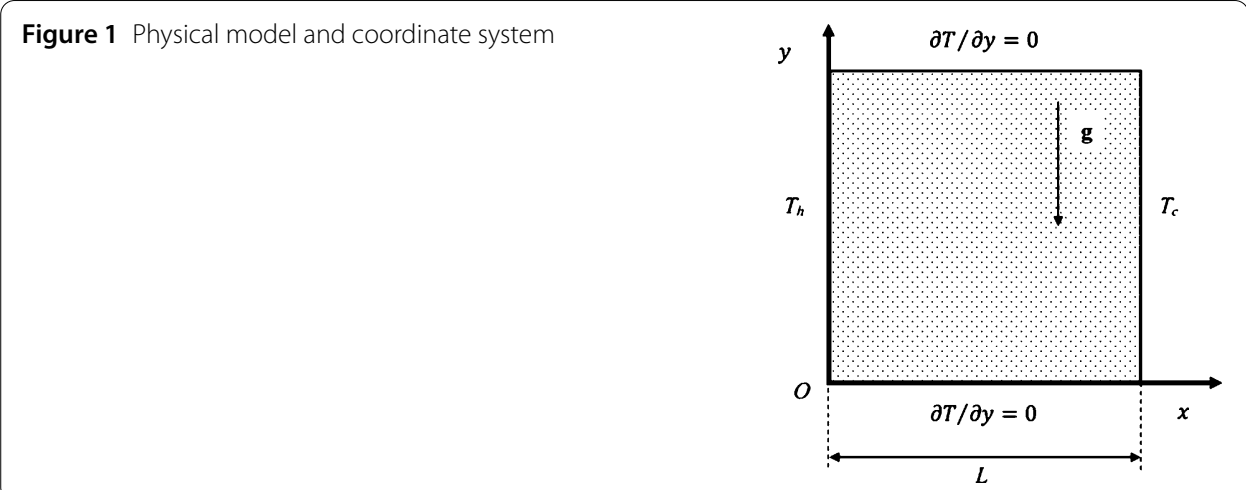


Equations (2.2)-(2.4) are written in Cartesian coordinates, and the pressure $p$ is eliminated by cross-differentiation. Introducing the stream function $\bar{\psi}$ defined by

$$
\bar{u}=\frac{\partial \bar{\psi}}{\partial \bar{y}}, \quad \bar{v}=-\frac{\partial \bar{\psi}}{\partial \bar{x}}
$$

we reduce equations (2.2) to (2.4) to

$$
\begin{aligned}
& \frac{\partial^{2} \bar{\psi}}{\partial \bar{x}^{2}}+\frac{\partial^{2} \bar{\psi}}{\partial \bar{y}^{2}}=-\frac{g K \rho_{0} \beta}{\mu} \frac{\partial T}{\partial \bar{y}}, \\
& \frac{\partial \bar{\psi}}{\partial \bar{y}} \frac{\partial T}{\partial \bar{x}}-\frac{\partial \bar{\psi}}{\partial \bar{x}} \frac{\partial T}{\partial \bar{y}}=\alpha_{m}\left(\frac{\partial^{2} T}{\partial \bar{x}^{2}}+\frac{\partial^{2} T}{\partial \bar{y}^{2}}\right),
\end{aligned}
$$

where $\alpha_{m}=k_{m} /(\rho c)_{f}$ is the effective thermal diffusivity. Introducing the dimensionless variables

$$
x=\frac{\bar{x}}{L}, \quad y=\frac{\bar{y}}{L}, \quad \psi=\frac{\bar{\psi}}{\alpha_{m}}, \quad \theta=\frac{T-T_{c}}{\Delta T} \quad \text { or } \quad \theta=\frac{T-T_{\infty}}{T_{f}-T_{\infty}}
$$

and substituting (2.8) into equations (2.6)-(2.7), we obtain:

$$
\begin{aligned}
& \frac{\partial^{2} \psi}{\partial x^{2}}+\frac{\partial^{2} \psi}{\partial y^{2}}=-R a \frac{\partial \theta}{\partial x}, \\
& \frac{\partial \psi}{\partial y} \frac{\partial \theta}{\partial x}-\frac{\partial \psi}{\partial x} \frac{\partial \theta}{\partial y}=\frac{\partial^{2} \theta}{\partial x^{2}}+\frac{\partial^{2} \theta}{\partial y^{2}},
\end{aligned}
$$

where $R a=g K \rho_{0} \beta \Delta T /\left(\alpha_{m} \mu\right)$ is the Rayleigh number, and $\Delta T=T_{h}-T_{c}$. Then we complete the mathematical model with the following corresponding boundary conditions:

$$
\begin{array}{ll}
\psi=0, & \frac{\partial \theta}{\partial x}=-B i(1-\theta) \quad \text { on } x=0, \\
\psi=0, & \theta=0 \quad \text { on } x=1, \\
\psi=0, & \frac{\partial \theta}{\partial y}=0 \quad \text { on } y=0,1,
\end{array}
$$

where $B i=L h_{f} / k_{f}$ is the Biot number. Note that for large values of $B i(\gg 1)$, from (2.11) it follows that $\theta(0)=1$ (isothermal left wall of the cavity).

The physical quantities of interest are the local and average Nusselt numbers $N u_{l}$ and $\overline{N u_{l}}$. The local Nusselt number at the left wall is given by

$$
N u_{l}=-\frac{\partial \theta}{\partial x}(0, y)=B i[1-\theta(0, y)]
$$

and the mean Nusselt number is

$$
\overline{N u}=-\left.\int_{0}^{1} \frac{\partial \theta}{\partial x}\right|_{x=0} d y=B i \int_{0}^{1}[1-\theta(0, y)] d y .
$$




\section{Numerical method}

The governing equations (2.9) and (2.10) coupled with the boundary conditions (2.11) are approximated numerically using the SADI (spline-alternating-direction-implicit) method, a combination of spline collocation and standard ADI (alternating-direction-implicit) methods. Each unknown is approximated by a cubic spline function. This is an efficient method, having good accuracy and low cost in implementation, as it leads to a governing linear tridiagonal system.

Using the fictitious time technique, we write equations (2.9) and (2.10) in the form

$$
\Phi_{i j}^{n+1 / 2}=F_{i j}^{n}+R_{i j}^{n} m_{\Phi, i j}^{n+1 / 2}+Q_{i j}^{n} M_{\Phi, i j}^{n+1 / 2}
$$

in the $x$-direction, with $m_{\Phi, i j}$ and $M_{\Phi, i j}$ denoting the spline approximations of the firstand second-order partial derivatives with respect to $x$. Then, in the $y$-direction, we write

$$
\Phi_{i j}^{n+1}=F_{i j}^{n+1 / 2}+R_{i j}^{n+1 / 2} l_{\Phi, i j}^{n+1 / 2}+Q_{i j}^{n+1 / 2} L_{\Phi, i j}^{n+1 / 2}
$$

where $l_{\Phi, i j}$ and $L_{\Phi, i j}$ are the spline approximations of $\left(\Phi_{y}\right)_{i j}$ and $\left(\Phi_{y y}\right)_{i j}$. In these equations, $i, j=0, N$ refer to the mesh nodes, $n$ denotes the false time step, and $\Phi_{i j}$ are the cubic spline approximations of the functions $\theta$ or $\psi$ at the grid nodes. The coefficients $F_{i j}, R_{i j}$, and $Q_{i j}$ at the two steps are given in Tables 1 and 2 . By the usual cubic spline collocation relations (see Pu and Kahawita [19]) we transform equations (3.1) and (3.2) to

$$
A_{i j} \varphi_{i-1, j}^{n+1 / 2}+B_{i j} \varphi_{i, j}^{n+1 / 2}+C_{i j} \varphi_{i+1, j}^{n+1 / 2}=D_{i j}, \quad i=1, \ldots, N-1,
$$

and

$$
A_{i j}^{\prime} \varphi_{i, j-1}^{n+1}+B_{i j}^{\prime} \varphi_{i, j}^{n+1}+C_{i j}^{\prime} \varphi_{i, j+1}^{n+1}=D_{i j}^{\prime}, \quad j=1, \ldots, N-1,
$$

respectively, where $\varphi$ can be the function $\Phi$ or one of its derivatives ( $m$ or $M$ for equation (3.3) and $l$ or $L$ for equation (3.4)). Obtaining two more equations from the boundary conditions, we get a closed tridiagonal, diagonally dominant linear system, which can be solved efficiently by the Thomas algorithm. For details on the mathematical computations,

Table 1 Coefficients in equation (3.3)

\begin{tabular}{llll}
\hline$\Psi$ & $F_{i j}^{n}$ & $R_{i j}^{n}$ & $Q_{i j}^{n}$ \\
\hline$\theta$ & $\theta_{i j}^{n}+\frac{\Delta t}{2}\left(\left.m_{\psi, i j}^{n}\right|_{\theta, i j} ^{n}+L_{\theta, i j}^{n}\right)$ & $-\frac{\Delta t}{2} l_{\psi, i j}^{n}$ & $\frac{\Delta t}{2}$ \\
$\psi$ & $\psi_{i j}^{n}+\frac{\Delta t}{2}\left(L_{\psi, i j}^{n}+R a m_{\theta, i j}^{n}\right)$ & 0 & $\frac{\Delta t}{2}$ \\
\hline
\end{tabular}

Table 2 Coefficients in equation (3.4)

\begin{tabular}{llll}
\hline$\Psi$ & $F_{i j}^{n+1 / 2}$ & $R_{i j}^{n+1 / 2}$ & $Q_{i j}^{n+1 / 2}$ \\
\hline$\theta$ & $\theta_{i j}^{n+1 / 2}+\frac{\Delta t}{2}\left(-I_{\psi, i j}^{n+1 / 2} m_{\theta, i j}^{n}+M_{\theta, i j}^{n}\right)$ & $\frac{\Delta t}{2} m_{\psi, i j}^{n+1 / 2}$ & $\frac{\Delta t}{2}$ \\
$\psi$ & $\psi_{i j}^{n+1 / 2}+\frac{\Delta t}{2}\left(M_{\psi, i j}^{n+1 / 2}+R a m_{\theta, i j}^{n}\right)$ & 0 & $\frac{\Delta t}{2}$ \\
\hline
\end{tabular}


see Rubin and Graves [20] or Pu and Kahawita [19]. The computations are iterated until a steady-state solution is reached, that is,

$$
\left\|\frac{\Phi^{n+1}-\Phi^{n}}{\Phi_{\max }^{n+1}}\right\|<10^{-7}
$$

where $n$ is the number of iterations.

When a steady-state solution is reached, the mean Nusselt number

$$
\overline{N u}=-\left.\int_{0}^{1} \frac{\partial \theta}{\partial x}\right|_{x=0} d y
$$

is approximated by the trapezoidal rule.

The procedure is described in more detail by Micula and Pop [21].

At the first step, in the $x$-direction, from time $n \Delta t$ to $(n+1 / 2) \Delta t$, discretizing equations (2.6)-(2.7), for every $i=1, \ldots, N-1$ and fixed $j \in\{0,1, \ldots, N\}$, we write

$$
\theta_{i j}^{n+1 / 2}=F_{\theta, i j}^{n}+R_{\theta, i j}^{n} m_{\theta, i j}^{n+1 / 2}+Q_{\theta, i j}^{n} M_{\theta, i j}^{n+1 / 2}
$$

and

$$
\psi_{i j}^{n+1 / 2}=F_{\psi, i j}^{n}+R_{\psi, i j}^{n} m_{\psi, i j}^{n+1 / 2}+Q_{\psi, i j}^{n} M_{\psi, i j}^{n+1 / 2}
$$

with the coefficients given in Table 1 . These equations are then put in the form

$$
A_{\theta, i j} \theta_{i-1 j}^{n+1 / 2}+B_{\theta, i j} \theta_{i j}^{n+1 / 2}+C_{\theta, i j} \theta_{i+1 j}^{n+1 / 2}=D_{\theta, i j}, \quad i=1, \ldots, N-1,
$$

and

$$
A_{\psi, i j} \psi_{i-1 j}^{n+1 / 2}+B_{\psi, i j} \psi_{i j}^{n+1 / 2}+C_{\psi, i j} \psi_{i+1 j}^{n+1 / 2}=D_{\psi, i j}, \quad i=1, \ldots, N-1
$$

respectively.

From the boundary conditions (2.11) we get another equation for $\theta_{i j}^{n+1 / 2}$,

$$
\theta_{N j}^{n+1 / 2}=0 .
$$

Also, combining the boundary conditions (2.11) with relations between cubic spline functions and their derivatives, we get one more equation

$$
A_{\theta, 0 j} \theta_{0 j}^{n+1 / 2}+B_{\theta, 0 j} \theta_{1 j}^{n+1 / 2}=D_{\theta, 0 j}
$$

The derivation of coefficients $A_{\theta, 0 j}, B_{\theta, 0 j}$, and $D_{\theta, 0 j}$ is given in the Appendix.

Equations (3.9), (3.11), and (3.12) form an $(N+1) \times(N+1)$ tridiagonal, diagonally dominant linear system in the unknowns $\left\{\theta_{i j}^{n+1 / 2}\right\}_{i=\overline{0, N}}$. When the system is solved and these values are found, the values of $\left\{m_{\theta, i j}^{n+1 / 2}\right\}_{i=\overline{0, N}}$ (and of $\left\{M_{\theta, i j}^{n+1 / 2}\right\}_{i=\overline{0, N}}$, if needed) can also be found. 
Similarly, for $\psi_{i j}^{n+1 / 2}$, equations (3.10) coupled with (from the boundary conditions)

$$
\psi_{0 j}^{n+1 / 2}=0, \quad \psi_{N j}^{n+1 / 2}=0
$$

form an $(N+1) \times(N+1)$ tridiagonal, diagonally dominant linear system for the unknowns $\left\{\psi_{i j}^{n+1 / 2}\right\}_{i=\overline{0, N}}$.

For the second step, in the $y$-direction, from time $(n+1 / 2) \Delta t$ to $(n+1) \Delta t$, we proceed similarly. For any fixed $i \in\{0,1, \ldots, N\}$ and for every $j=1, \ldots, N-1$, using the coefficients from Table 2, we get equations

$$
A_{\theta, i j}^{\prime} l_{\theta, i j-1}^{n+1}+B_{\theta, i j}^{\prime} l_{\theta, i j}^{n+1}+C_{\theta, i j}^{\prime} l_{\theta, i j+1}^{n+1}=D_{\theta, i j}^{\prime}, \quad j=1, \ldots, N-1
$$

and

$$
A_{\psi, i j}^{\prime} \psi_{i j-1}^{n+1}+B_{\psi, i j}^{\prime} \psi_{i j}^{n+1}+C_{\psi, i j}^{\prime} \psi_{i j+1}^{n+1}=D_{\psi, i j}^{\prime}, \quad j=1, \ldots, N-1 .
$$

Again, from the boundary conditions (2.11) we get two more equations for $l_{\theta, i j}^{n+1}$,

$$
l_{\theta, i 0}^{n+1}=0, \quad l_{\theta, i N}^{n+1}=0,
$$

and two for $\psi_{i j}^{n+1}$

$$
\psi_{i 0}^{n+1}=0, \quad \psi_{i N}^{n+1}=0 .
$$

Then equations (3.14) and (3.16) form a closed linear system for the unknowns $\left\{l_{\theta, i j}^{n+1}\right\}_{j=\overline{0, N}}$ (again, from these the values of $\left\{\theta_{i j}^{n+1}\right\}_{i=\overline{0, N}}$ and $\left\{L_{\theta, i j}^{n+1}\right\}_{j=\overline{0, N}}$ can also be found). Similarly, equations (3.15) and (3.17) give an $(N+1) \times(N+1)$ tridiagonal linear system for the unknowns $\left\{\psi_{i j}^{n+1}\right\}_{j=\overline{0, N}}$.

\section{Numerical results and discussion}

We apply the SADI method to our problem taking the values $R a=10,100,1000$ and $B i=$ $0.5,5,50,100$. The method is validated by comparing the values of $\overline{N u}$ we obtained with other well-known results in the literature (see Table 3).

We use uniform meshes of $N \times N$ nodes for $N=100,200,300$, and 400. A sensitivity to the grid size test is performed, and the results are seen in Table 4. As the values in

Table 3 Comparison of the average Nusselt number $\overline{N u}$ at the hot wall

\begin{tabular}{lllll}
\hline Authors & \multicolumn{4}{c}{ Ra } \\
\cline { 2 - 5 } & 10 & 100 & 1000 & 10,000 \\
\hline Walker and Homsy [22] & - & 3.097 & 12.960 & 51.000 \\
Bejan [23] & - & 4.200 & 15.800 & 50.800 \\
Beckermann et al. [24] & - & 3.113 & - & 48.900 \\
Gross et al. [13] & - & 3.141 & 13.448 & 42.583 \\
Moya et al. [25] & 1.065 & 2.801 & - & - \\
Manole and Lage [26] & - & 3.118 & 13.637 & 48.117 \\
Baytas and Pop [27] & 1.079 & 3.160 & 14.060 & 48.330 \\
Sheremet and Pop [28] & 1.071 & 3.104 & 13.839 & 49.253 \\
Present results (unif. grid) & 1.079 & 3.111 & 13.602 & - \\
Present results (nonunif. grid) & 1.079 & 3.113 & 13.650 & 48.836 \\
\hline
\end{tabular}


Table 4 Variation of $\overline{N u}$ with the grid, $R a=1000, B i=100$, uniform grid

\begin{tabular}{lll}
\hline Grid & $\overline{N u}$ & $\frac{\left|\overline{N u}_{i \times j}-\overline{N u}_{400} \times 400\right|}{|\overline{N u} i \times j|} \times 100 \%$ \\
\hline $100 \times 100$ & 11.499 & 0.86 \\
$200 \times 200$ & 11.581 & 0.15 \\
$300 \times 300$ & 11.594 & 0.03 \\
$400 \times 400$ & 11.598 & - \\
\hline
\end{tabular}

Table 5 Variation of $\overline{N u}$ with $B i$, for $R a=10,100,1000$, uniform grid

\begin{tabular}{|c|c|c|}
\hline$R a$ & $B i$ & $\overline{\mathrm{Nu}}$ \\
\hline 10 & $\begin{array}{c}0.5 \\
5 \\
50 \\
100 \\
\boldsymbol{\theta}=\mathbf{1}\end{array}$ & $\begin{array}{l}0.3347 \\
0.8761 \\
1.0548 \\
1.0668 \\
\mathbf{1 . 0 7 9 1}\end{array}$ \\
\hline 100 & $\begin{array}{c}0.5 \\
5 \\
50 \\
100 \\
\boldsymbol{\theta}=\mathbf{1}\end{array}$ & $\begin{array}{l}0.3737 \\
1.6836 \\
2.8732 \\
2.9892 \\
\mathbf{3 . 1 1 0 6}\end{array}$ \\
\hline 1000 & $\begin{array}{c}0.5 \\
5 \\
50 \\
100 \\
\boldsymbol{\theta}=\mathbf{1}\end{array}$ & $\begin{array}{r}0.4427 \\
3.1415 \\
10.0772 \\
11.5979 \\
\mathbf{1 3 . 6 0 1 7}\end{array}$ \\
\hline
\end{tabular}



$R a=10$

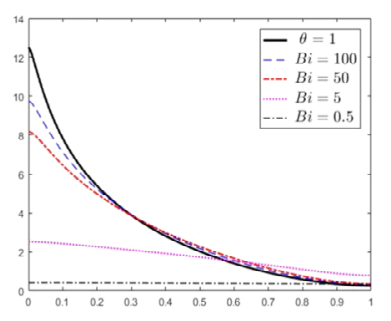

$R a=100$

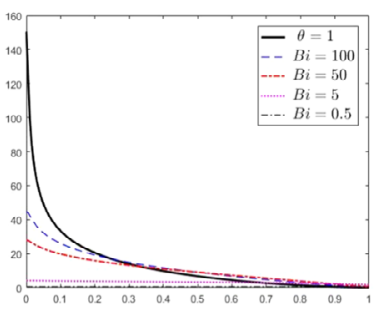

$R a=1000$

Figure 2 Variation of local Nusselt number with $B$ i for $R a=10,100,1000$

the table show, the results vary little from $100 \times 100$ to $400 \times 400$ meshes. Therefore, for various values of the parameters $R a$ and $B i$, in our calculations, we considered $200 \times 200$ grids. The values of the average Nusselt number $\overline{N u}$ are presented in Table 5 . For each value of $R a$, note that as the values of $B i$ become larger, the results approach the values corresponding to the case where the boundary condition is $\theta=1$ on the left wall of the cavity.

Also, the average Nusselt number increases as the values of the Biot number increase, as can be seen from Table 5 .

Figure 2 shows the variation of the local Nusselt number with $B i$, again the tendency toward the case $\theta=1$ on $x=0$ being evident. In Figs. $3-5$ the streamlines and isotherms are depicted for all the values of the parameters $R a$ and $B i$ considered.

For the maximum values of the parameters $(R a=1000$ and $B i=100)$, we also performed calculations using nonuniform grids. We used a nonuniform mesh obtained by keeping the 


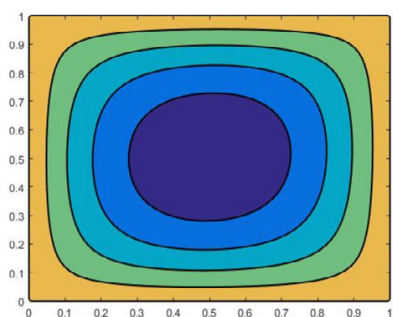

$B i=0.5$

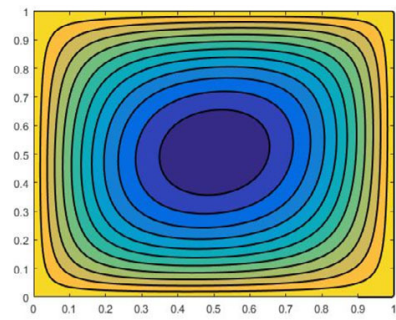

$B i=5$



$B i=\mathbf{5 0}$

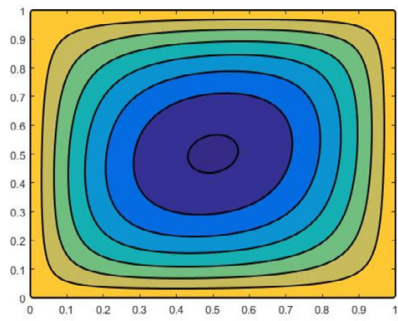

$B i=100$

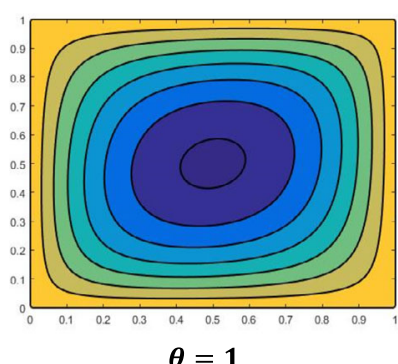



$B i=0.5$

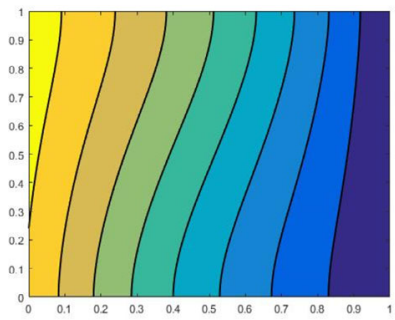

$B i=5$



$B \boldsymbol{i}=\mathbf{5 0}$

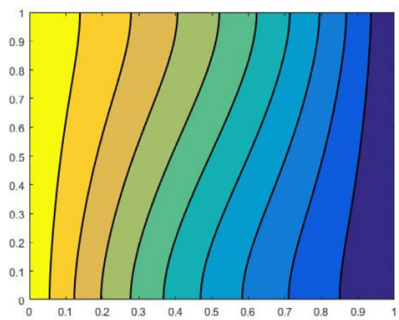

$B i=100$

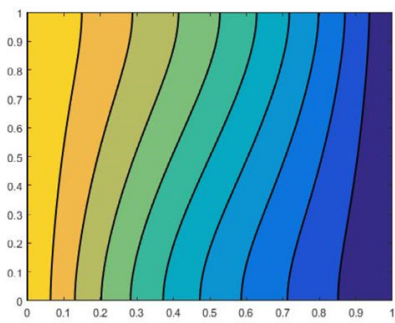

$\theta=1$

Figure 3 Streamlines (first column) and isotherms (second column), $R a=10$

spacing ratio from the wall to the center $h_{i+1} / h_{i}$ a constant (strictly greater than 1 ), which depends on the desired number of nodes. In this case, we produced a symmetric grid with $h_{\min } \approx 5 \times 10^{-5}$ (near the walls) and $h_{\max } \approx 0.02$ (at the center). The variation of $\overline{N u}$ with the number of nodes for a nonuniform grid is shown in Table 6 . As it can be seen, there are insignificant differences from smaller to larger numbers of nodes. Note that the values 

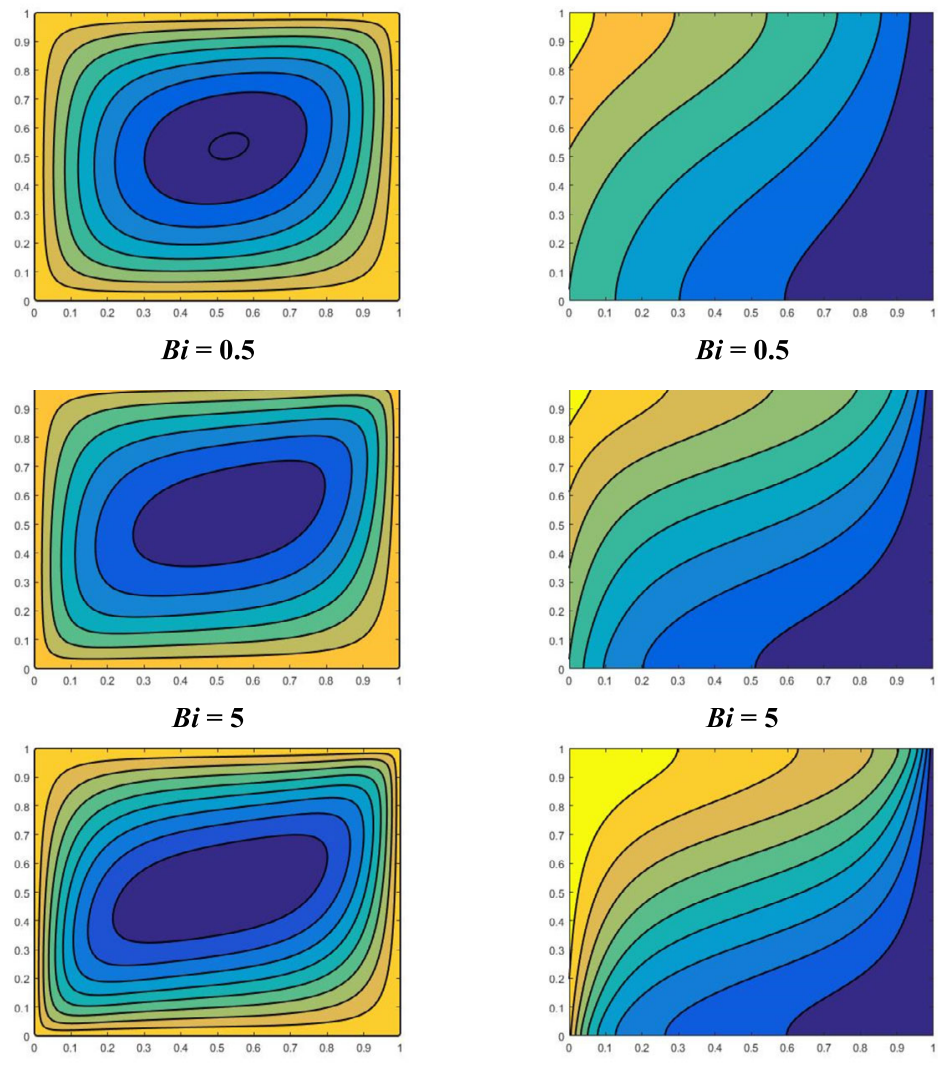

$B i=\mathbf{5 0}$
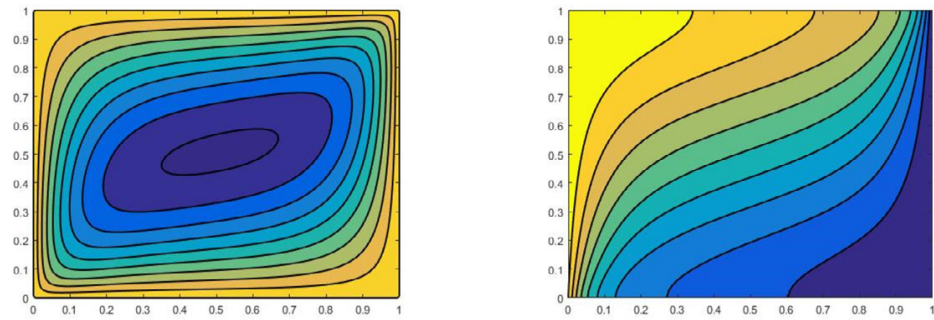

$B i=100$
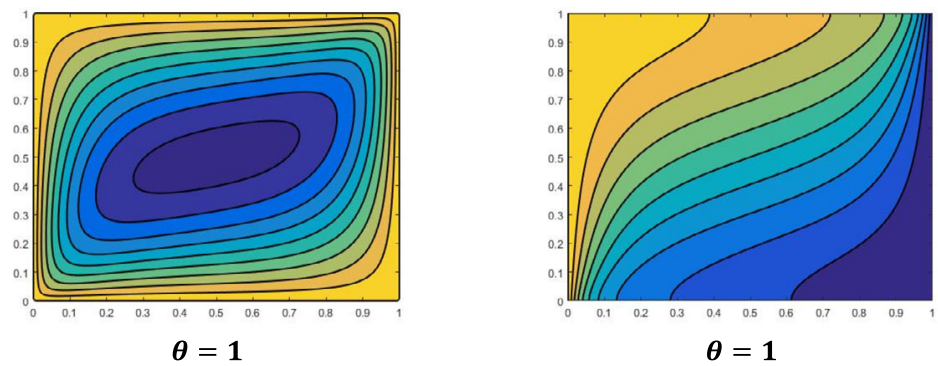

Figure 4 Streamlines (first column) and isotherms (second column), $R a=100$

obtained when a nonuniform mesh was used are slightly higher than those obtained by a uniform grid at the same number of nodes. That was also evident in the simple case of a hot wall, $\theta=1$, on $x=0$ (see Table 3). 

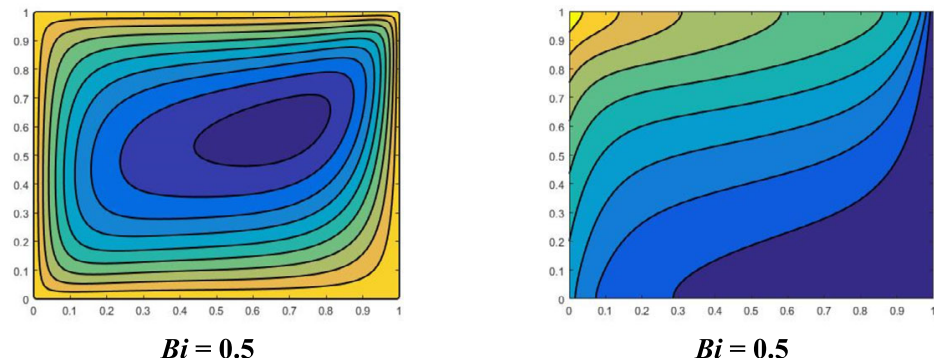

$B i=0.5$
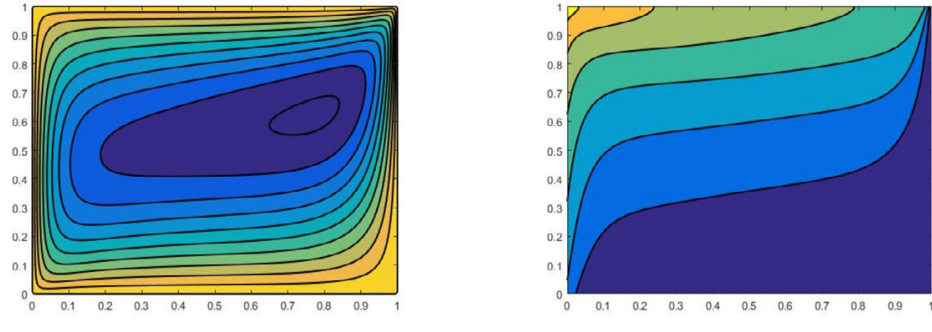

$B i=5$


$B i=\mathbf{5 0}$
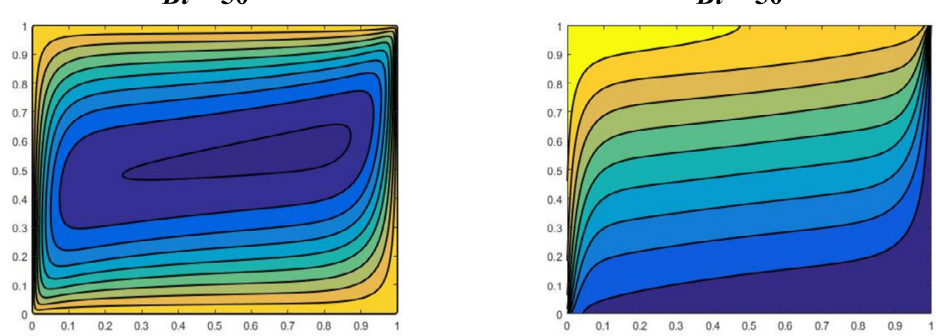

$B i=100$
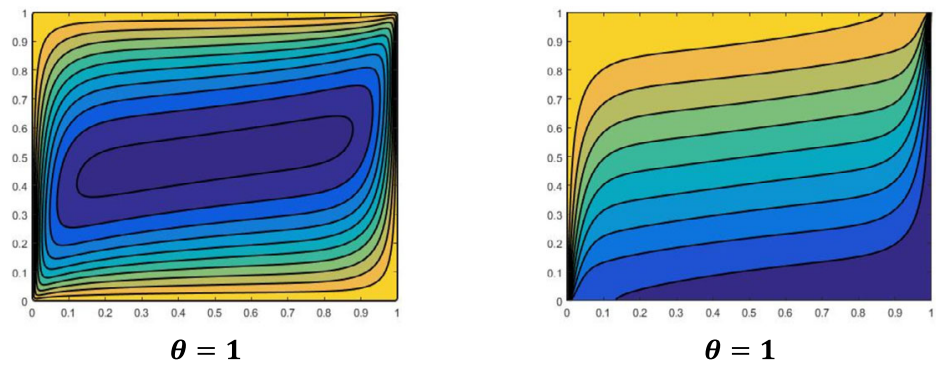

Figure 5 Streamlines (first column) and isotherms (second column), $R a=1000$

\section{Conclusions}

We used a cubic spline collocation scheme to obtain the numerical solution of the problem of free convection inside a porous square cavity with convective boundary condition. This is an efficient method as it leads to a sparse (tridiagonal) linear system that can be solved faster using the Thomas algorithm. The SADI method is especially useful when 
Table 6 Variation of $\overline{N u}$ with the grid, $R a=1000, B i=100$, nonuniform grid

\begin{tabular}{lll}
\hline Grid & $\overline{N u}$ & $\frac{\left|\overline{N u}_{i \times j} \overline{N u}_{300} \times 300\right|}{\left|\overline{N u}_{i j j}\right|} \times 100 \%$ \\
\hline $70 \times 70$ & 11.722 & 0.80 \\
$100 \times 100$ & 11.643 & 0.13 \\
$200 \times 200$ & 11.630 & 0.02 \\
$300 \times 300$ & 11.628 & - \\
\hline
\end{tabular}

the boundary conditions involve values of the derivatives, not just of the function, as it is the case here. That is because the governing linear system can be written for the values of the (first or second) derivatives at the nodes, not just for the functional values, as in other classical methods. This can be chosen by convenience (depending on the boundary conditions), with fewer extra computations and without other local numerical differentiation techniques. This saves computational time and storage space. The method is second-order accurate, even when nonuniform meshes are used, as is often necessary near the boundary. At the same time, it yields accurate results, in good agreement with other reported values, as Table 3 shows.

As the numerical results and graphs show, when the values of $B i$ increase $(\gg 1)$, the boundary condition on the left wall approaches the case $\theta=1$ (isothermal left wall of the cavity).

The Biot number enhances the release of heat energy, substantially cooling the system. This analysis may certainly have practical impact on obtaining the conditions needed to design an integrated system to achieve optimal functionality.

\section{Appendix}

In this section, we derive equation (3.12). To simplify the writing, since this is only done for the function $\theta$ at the first step, we omit the subscript $\theta$ for the coefficients and the superscript $n+1 / 2$ for the function. Also, since $j \in\{0,1, \ldots, N\}$ is fixed, we also omit it. The notations $m$ and $M$ for the approximations of the first and second derivatives of $\theta$ with respect to $x$ are also accordingly simplified. So, we want to derive the equation of the form

$$
A_{0} \theta_{0}+B_{0} \theta_{1}=D_{0}
$$

From discretization (3.7) we get (for $i=0$ and $i=1$ )

$$
\theta_{0}=F_{0}+R_{0} m_{0}+Q_{0} M_{0}
$$

and

$$
\theta_{1}=F_{1}+R_{1} m_{1}+Q_{1} M_{1}
$$

From the boundary conditions (2.11) we also have

$$
m_{0}=-B i\left(1-\theta_{0}\right)
$$


By the basic cubic spline relations between functional and derivative values (see Rubin and Graves [17]) we know that

$$
m_{1}-m_{0}=\frac{h_{1}}{2}\left(M_{0}+M_{1}\right)
$$

and

$$
M_{1}=\frac{2}{h_{1}}\left(m_{0}+2 m_{1}-\frac{3}{h_{1}}\left(\theta_{1}-\theta_{0}\right)\right) .
$$

We eliminate four of the six unknowns, namely $m_{0}, M_{0}, m_{1}$, and $M_{1}$ from five equations (A.2)-(A.6) to obtain a linear relationship between the last two remaining unknowns $\theta_{0}$ and $\theta_{1}$.

Denoting

$$
K=Q_{1}+\frac{h_{1}}{2} R_{1}, \quad J=\frac{K+Q_{1}}{Q_{0}},
$$

we obtain equation (A.1) with

$$
\begin{aligned}
& A_{0}=B i\left(\frac{4}{h_{1}} K+\frac{2}{h_{1}} Q_{1}-R_{0} J\right)+\frac{6}{h_{1}^{2}} K+J, \\
& B_{0}=1-\frac{6}{h_{1}^{2}} K, \\
& D_{0}=B i\left(\frac{4}{h_{1}} K+\frac{2}{h_{1}} Q_{1}-R_{0} J\right)+F_{0} J+F_{1} .
\end{aligned}
$$

\section{Acknowledgements}

The authors wish to express their thanks to Dr. Teodor Groşan for reading the paper.

\section{Funding}

There is no funding for this paper.

\section{Abbreviations}

g, acceleration vector due to gravity; $K$, permeability of the porous medium; $L$, height of the cavity; $N u_{x}$, local Nusselt number; $\overline{N u}$, average Nusselt number; $p$, pressure; $R a$, Rayleigh number for porous medium; $B i$, Biot number; $T$, temperature; $T_{c}$, temperature of the cooled wall; $T_{h}$, temperature of the left hot wall; $v$, velocity vector; $\bar{u}, \bar{v}$, dimensional velocity component in the $\bar{x}$ - and $\bar{y}$-axes; $\bar{x}, \bar{y}$, dimensional Cartesian coordinates; $x, y$, dimensionless Cartesian coordinates; $\alpha_{m}$, thermal diffusivity of the porous medium; $\beta$, coefficient of thermal expansion of the nanofluid; $\varepsilon$, porosity; $\theta$, dimensionless temperature; $\mu$, dynamic viscosity of the fluid; $\rho_{0}$, density of the fluid; $\bar{\psi}$, dimensional stream function; $\psi$, dimensionless stream function.

\section{Availability of data and materials}

The data that support the findings of this study are available within the paper.

\section{Competing interests}

The authors declare that they have no competing interests.

\section{Authors' contributions}

Both authors contributed equally and significantly in writing this paper. SM has obtained the numerical results and contributed to the discussion of the results, and IP has formulated the problem and made some discussion. Both authors read and approved the final manuscript.

\section{Publisher's Note}

Springer Nature remains neutral with regard to jurisdictional claims in published maps and institutional affiliations. 


\section{References}

1. Ingham, D.B., Pop, I. (eds.): Transport Phenomena in Porous Media Pergamon, Oxford (1998)

2. Ingham, D.B., Pop, I. (eds.): Transport Phenomena in Porous Media, Vol. II Pergamon, Oxford (2002)

3. Ingham, D.B., Pop, I. (eds.): Transport Phenomena in Porous Media, Vol. III Elsevier, Oxford (2005)

4. Pop, I., Ingham, D.B.: Convective Heat Transfer: Mathematical and Computational Modeling of Viscous Fluids and Porous Media. Pergamon, Oxford (2001)

5. Kaviany, M.: Principals of Heat Transfer in Porous Media, 2nd edn. Springer, New York (1999)

6. Vafai, K. (ed.): Handbook of Porous Media 2nd edn. Taylor \& Francis, New York (2005)

7. Vafai, K.: Porous Media: Applications in Biological Systems and Biotechnology. CRC Press, Tokyo (2010)

8. Vadasz, P. (ed.): Emerging Topics in Heat and Mass Transfer in Porous Media Springer, New York (2008)

9. Bejan, A.: Convection Heat Transfer, 4th edn. Wiley, New York (2013)

10. Nield, D.A., Bejan, A.: Convection in Porous Media, 5th edn. Springer, New York (2017)

11. Bear, J.: Modeling Phenomena of Flow and Transport in Porous Media. Springer, New York (2018)

12. Prasad, V., Kulacki, F.A.: Convective heat transfer in a rectangular porous cavity-effect of aspect ratio on flow structure and heat transfer. J. Heat Transf. 106, 158-165 (1984)

13. Gross, R.J., Bear, M.R., Hickox, C.E.: The application of flux-corrected transport (FCT) to high Rayleigh number natural convection in a porous medium. In: Proceedings of the 8th International Heat Transfer Conference, San Francisco (1986)

14. Chaudhary, R.C., Jain, P.: Unsteady free convection boundary layer flow past an impulsively started vertical surface with Newtonian heating. Rom. J. Phys. 51, 911-925 (2006)

15. Abdelwahed, M., Chorfi, N., Malek, R.: Reconstruction of Tesla micro-valve using topological sensitivity analysis. Adv. Nonlinear Anal. 9, 567-590 (2020)

16. Abdelwahed, M., Chorfi, N.: On the convergence analysis of a time dependent elliptic equation with discontinuous coefficients. Adv. Nonlinear Anal. 9, 1145-1160 (2020)

17. Chorfi, N., Abdelwahed, M., Berselli, L.C.: On the analysis of a geometrically selective turbulence model. Adv. Nonlinear Anal. 9, 1402-1419 (2020)

18. Aziz, A.: A similarity solution for laminar thermal boundary layer over a flat plate with a convective surface boundary condition. Commun. Nonlinear Sci. Numer. Simul. 14, 1064-1068 (2009)

19. Pu, W., Kahawita, R.: The numerical solution of the unsteady natural convection flow in a square cavity at high Rayleigh number using SADI method. Appl. Math. Mech. 8, 219-228 (1987)

20. Rubin, G., Graves, R.A.: A cubic spline approximation for problems in fluid mechanics. National Aeronautics and Space Administration (1975) Technical Report

21. Micula, S., Pop, I.: Numerical results for the classical free convection flow problem in a square porous cavity using spline functions. Int. J. Numer. Methods Heat Fluid Flow 31(3), 753-765 (2021) https://doi.org/10.1108/HFF-03-2020-0159

22. Walker, K.L., Homsy, G.M.: Convection in a porous cavity. J. Fluid Mech. 87, 449-474 (1978)

23. Bejan, A.: On the boundary layer regime in a vertical enclosure filled with a porous medium. Lett. Heat Mass Transfer 6, 93-102 (1979)

24. Beckermann, C., Viskanta, R., Ramadhyani, S.: A numerical study of non-Darcian natural convection in a vertical enclosure filled with a porous medium. Numer. Heat Transf. 10, 557-570 (1987)

25. Moya, S.L., Ramos, E., Sen, M.: Numerical study of natural convection in a tilted rectangular porous material. Int. J. Heat Mass Transf. 30(4), 741-756 (1987)

26. Manole, D.M., Lage, J.L.: Numerical benchmark results for natural convection in a porous medium cavity. In: Heat and Mass Transfer in Porous Media. ASME Conference, vol. 105, pp. 44-59 (1992)

27. Baytas, A.C., Pop, I.: Free convection in oblique enclosures filled with a porous medium. Int. J. Heat Mass Transf. 42(6), 1047-1057 (1999)

28. Sheremet, M.A., Pop, I.: Natural convection in a square porous cavity with sinusoidal temperature distributions on both side walls filled with a nanofluid: Buongiorno's mathematical model. Transp. Porous Media 105, 411-429 (2014)

\section{Submit your manuscript to a SpringerOpen ${ }^{\circ}$ journal and benefit from:}

- Convenient online submission

- Rigorous peer review

- Open access: articles freely available online

- High visibility within the field

- Retaining the copyright to your article

Submit your next manuscript at $>$ springeropen.com 